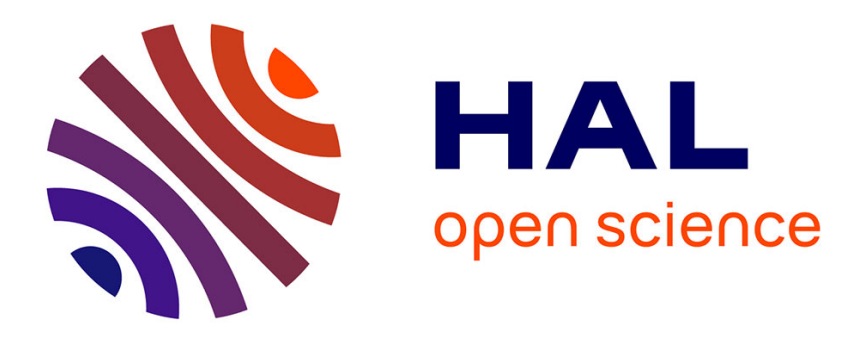

\title{
Quelques réflexions méthodologiques sur les abandons de sanctuaires en Grèce antique
}

Alaya Palamidis

\section{To cite this version:}

Alaya Palamidis. Quelques réflexions méthodologiques sur les abandons de sanctuaires en Grèce antique. Revue de l'histoire des religions, 2018, Un dieu peut-il mourir? Enquête sur la fin des cultes dans l'Antiquité gréco-romaine, 235, pp.255-273. 10.4000/rhr.8904 . hal-02197273v2

\section{HAL Id: hal-02197273 \\ https://hal.science/hal-02197273v2}

Submitted on 2 Aug 2021

HAL is a multi-disciplinary open access archive for the deposit and dissemination of scientific research documents, whether they are published or not. The documents may come from teaching and research institutions in France or abroad, or from public or private research centers.
L'archive ouverte pluridisciplinaire HAL, est destinée au dépôt et à la diffusion de documents scientifiques de niveau recherche, publiés ou non, émanant des établissements d'enseignement et de recherche français ou étrangers, des laboratoires publics ou privés. 


\section{Revue}

\section{de I'histoire des religions}

\section{Un dieu peut-il mourir ? \\ Enquête sur la fin des cultes dans l'Antiquité gréco-romaine}

\section{Can Gods die? \\ Investigating the Ends of Cults in Greco-Roman Antiquity}

Karin Mackowiak, Christian Stein, Avant-propos - Nicole BeLAYCHE, Les discours chrétiens sur la « fin des cultes » publics au Levant : l'argument des sanctuaires — Françoise VAN HAEPEREN, Fin des temples et fin des cultes à Ostie : une histoire complexe - Alaya Palamidis, Quelques réflexions méthodologiques sur les abandons de sanctuaires en Grèce antique — Lorenz BAUMER, Raisons de l'abandon et du maintien de sanctuaires ruraux en Attique : quelques cas d'étude - Perrine Kossmann, Disparition de la dynastie, extinction du culte ? Le cas des Lagides - Karin MaCkOWIAK, Hagnon et Brasidas à Amphipolis : chronique d'une « fin de culte » annoncée ? - Blaise Pichon, La fin des cultes et des sanctuaires païens urbains en Belgique et en Lyonnaise (III ${ }^{\mathrm{e}} \mathrm{s}$. - début du Ve apr. J.-C.) - Michel HumM, La « barbarisation » de Poséidonia et la fin des cultes grecs à Paestum. 


\section{Quelques réflexions méthodologiques sur les abandons de sanctuaires en Grèce antique}

En raison du nombre très limité de sources écrites concernant la disparition de cultes en Grèce antique, ainsi que les difficultés importantes que pose leur interprétation, l'étude de ce phénomène est presque exclusivement possible grâce à l'analyse des données des fouilles archéologiques. Pourtant, l'identification de l'abandon d'un sanctuaire est souvent délicate, en raison notamment des conditions de fouilles mais également des processus post-dépositionnels qui peuvent entraîner une destruction des couches stratigraphiques supérieures. De plus, le culte d'un sanctuaire abandonné peut être transféré dans un nouveau sanctuaire, si bien que les sources archéologiques ne peuvent suffire à attester l'interruption d'un culte.

\section{Some methodological thoughts on the abandonment of sanctuaries in ancient Greece}

Given the limited number of written sources about the disappearance of cults in ancient Greece, as well as the important difficulties raised by their interpretation, the study of this phenomenon is almost exclusively possible through the analysis of the information obtained from archaeological excavations. However, identifying the abandonment of a sanctuary is often difficult, mainly because of the excavation conditions but also because of post-depositional processes that may cause the destruction of the upper stratigraphic layers. Moreover, the cult of an abandoned sanctuary may have been transferred to a new sanctuary, so that the archaeological sources cannot be sufficient to attest to the disappearance of a cult. 
Comment identifier des disparitions de cultes dans le monde grec, lorsqu'elles ne sont pas liées à la concurrence du christianisme? Les textes qui mentionnent de telles disparitions sont extrêmement rares et d'interprétation difficile. Ainsi, Hérodote relate la décision de Clisthène, tyran archaïque de Sicyone, de remplacer le culte du héros Adraste par celui de Mélanippe. L'historien précise cependant que l'hérôon d'Adraste est encore visible à son époque ${ }^{1}$ : faut-il dès lors supposer une continuité du culte d'Adraste ? Un passage de l'œuvre de Thucydide semble se référer à un épisode similaire, l'abandon du culte d'Hagnon, l'œciste d'Amphipolis, au profit de celui du général lacédémonien Brasidas au cours de la guerre du Péloponnèse ; dans ce cas, c'est la réalité même du culte d'Hagnon qui est débattue parmi les chercheurs².

Dès lors, toute étude des disparitions de cultes est nécessairement une étude des abandons de sanctuaires. Quelques sources écrites se réfèrent à de tels abandons. Strabon nous apprend par exemple que le sanctuaire de Dindymène dans l'ancienne Magnésie du Méandre n'existe plus à son époque du fait du déplacement de la ville; le géographe emploie par ailleurs l'imparfait pour désigner l'Héraion de Pérachora, qui n'a effectivement livré aucune trace de fréquentation pour le début de l'époque impériale ${ }^{3}$. D'autres textes sont toutefois plus ambigus. Pausanias décrit ainsi de nombreux lieux de culte en ruines et précise, dans le cas du sanctuaire d'Aphrodite Ourania à Olympie et dans celui d'Apollon Pythios près de Phénéos, que des sacrifices ont encore lieu parmi les vestiges ${ }^{4}$. Ces précisions permettent-elles de conclure à une extinction du culte dans les autres sanctuaires détruits ? Ou le Périégète manque-t-il simplement d'informations à

1. Hérodote, V, 67.

2. Thucydide, $\mathrm{V}, 11,1$. Voir par exemple Manuela Mari, «Amphipolis Between Athens and Sparta. A Philological and Historical Commentary on Thuc. V 11, 1 », Mediterrenao Antico, t. 15, fasc. 1-2, p. 327-353, en particulier p. 342-344 et la contribution de Karin Mackowiak dans ce volume.

3. Strabon, XIV, 1, 40 (C 647); VIII, 6, 22 (C 380). Voir infra à propos de l'abandon de l'Héraion de Pérachora.

4. Pausanias, VI, 20, 6 (Olympie) ; VIII, 15, 5 (Phénéos). Voir William Kendrick Pritchett, Pausanias Periegetes t. 2, Amsterdam, J.C. Gieben, 1999, p. 215-216 pour une liste de temples en ruines chez Pausanias. 
leur sujet ? Plutarque consacre quant à lui un traité à la disparition des oracles, en particulier en Béotie. Il cite notamment l'oracle du Ptoion, sanctuaire pourtant attesté tout au long de l'époque impériale $^{5}$. Bien que cet exemple ne signifie pas nécessairement que tous les sanctuaires qu'il nomme sont encore fréquentés lors de la rédaction du traité, l'auteur semble s'intéresser uniquement à la fonction oraculaire des sanctuaires béotiens, et n'apporte ainsi aucune information sur une éventuelle interruption du culte. Une inscription également invoquée dans les études concernant les

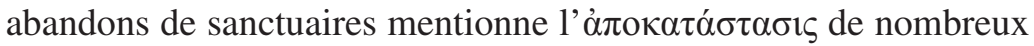
lieux de culte attiques ${ }^{6}$. Ce terme, que l'on peut traduire par « restauration », est toutefois équivoque puisqu'il peut désigner à la fois une refondation et une réparation des sanctuaires concernés ; en raison du mauvais état de conservation de la pierre, son sens exact ne peut être déterminé. De la même façon, les interruptions de fêtes attestées par l'épigraphie ${ }^{7}$ n'impliquent pas nécessairement un abandon des sanctuaires mais uniquement une diminution de la fréquence et du faste des activités cultuelles.

En raison de ces difficultés, ce sont principalement les sources archéologiques qui permettent l'étude des abandons de sanctuaires et de cultes. Susan Alcock et Lorenz Baumer se sont ainsi intéressés à la désaffection des lieux de culte ruraux dans un cadre régional à partir des résultats de fouilles et de prospections de surface ${ }^{8}$. Deux questions, que cet article se propose de mettre en évidence,

5. Plutarque, Moralia, en particulier $411 \mathrm{f}-412 \mathrm{~d}$. Voir notamment les inscriptions $I G$ VII, 2712; 2713, 1. 49-50 et 57 ; 2726; 4150-4152; SEG 15, 330, 1. 12, d'époque impériale, qui proviennent du Ptoion ou mentionnent le sanctuaire, ainsi que Pausanias, IX, 23, 6 .

6. $I G \mathrm{II}^{2}, 1035$. Sur cette inscription, voir notamment Gerald Culley, « The Restoration of Sanctuaries in Attica : IG II ${ }^{2}, 1035 »$, Hesperia, t. 44, fasc. 2, 1975, p. 207-223 ; « The Restoration of Sanctuaries in Attica, II : The Structure of $I G$ II $^{2}$, 1035 and the Topography of Salamis », Hesperia, t. 46, fasc. 3, 1977, p. 282-298; Geoffrey Schmalz, «Inscribing a Ritualized Past : the Attic Restoration Decree

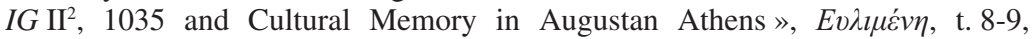
2007-2008, p. 9-46.

7. Voir notamment Nadine Deshours, L'été indien de la religion civique, Bordeaux, Ausonius Éditions, 2011, p. 97-129 (époque hellénistique) ; Stephan Hotz, «Ritual Traditions in the Discourse of the Imperial Period», Ritual and Communication in the Graeco-Roman World, dir. Eftychia Stavrianopoulou, Liège, CIERGA, 2006, p. 283-296.

8. Susan E. Alcock, « Minding the Gap in Hellenistic and Roman Greece », Placing the Gods. Sanctuaries and Sacred Space in Ancient Greece, dir. S.E. Alcock, Robin Osborne, Oxford, Clarendon Press, 1996, p. 247-261 ; 
doivent cependant être prises en compte. Premièrement, l'identification de l'abandon d'un sanctuaire n'est pas toujours certaine. La réutilisation de son espace ou l'identification d'un rituel d'abandon attestent parfois qu'un lieu de culte n'est plus fréquenté, mais l'absence, à partir d'une certaine date, de matériel pouvant être mis en lien avec le culte constitue souvent l'unique indice d'un abandon. Pourtant, des processus post-dépositionnels ou l'impossibilité de réaliser des campagnes de fouilles sur l'ensemble de la surface d'un lieu de culte peuvent expliquer l'existence de "faux négatifs », c'est-à-dire de sanctuaires qui ne produisent aucun matériel archéologique correspondant à une période de fréquentation du lieu de culte. Il est donc nécessaire, à défaut d'indices supplémentaires suggérant un abandon, d'étudier la topographie d'un site et son histoire postérieure, jusqu'au moment des fouilles, pour évaluer la signification de l'absence de matériel.

La seconde question est liée à l'apport même des sources archéologiques pour l'étude des disparitions de cultes : l'abandon d'un sanctuaire est-il synonyme de l'abandon du culte de la ou des divinités honorées à cet endroit ? Quelques rares sources écrites nous apprennent qu'il est possible de transférer un culte, c'est-à-dire de continuer à honorer une divinité à un autre emplacement après l'abandon de son sanctuaire. S'agit-il cependant de phénomènes exceptionnels ou faut-il supposer que tout sanctuaire abandonné est susceptible d'être concerné par un tel transfert? À travers une analyse des éléments permettant l'identification des abandons de sanctuaires puis des transferts de cultes, nous interrogerons les informations relatives à la disparition de cultes qu'il est possible de déduire des vestiges matériels des sanctuaires.

Lorenz Baumer, Mémoires de la religion grecque, Paris, Le Cerf, 2010, p. $47-84$. 


\section{L'IDENTIFICATION DE L'ABANDON D'UN SANCTUAIRE}

\section{La désacralisation des sanctuaires}

Les fouilles archéologiques ont révélé, dans certains sanctuaires, la construction d'édifices antiques qui ne semblent pas liés au fonctionnement du lieu de culte. Dans l'Héraion de Pérachora, un grand bâtiment est par exemple construit à l'emplacement de la «West Court» à l'époque impériale 9 . Sa fonction domestique et agricole est indiquée par la présence d'un four, d'un pressoir ainsi que, à proximité immédiate, d'une tombe contemporaine. La construction de cet édifice est-elle une conséquence de l'abandon du sanctuaire? La surface d'un sanctuaire peut être réduite sans que cela n'entraîne une interruption de sa fréquentation, comme le montre par exemple le cas du «stele shrine» sur l'agora de Corinthe : la partie Est de cet enclos carré est en effet recouverte par une partie de la stoa Sud vers la fin du Iv ${ }^{\text {e }}$ siècle av. J.-C., mais le culte est encore attesté pendant plusieurs décennies ${ }^{10}$. D'autres éléments indiquent cependant que la construction de la maison à Pérachora est postérieure à l'abandon du sanctuaire. Les traces d'incendie du « West Court » et de la « stoa en $\mathrm{L} »^{11}$ suggèrent une destruction au moment du sac de Corinthe par Lucius Mummius en 146 av. J.-C. L'absence de matériel postérieur au $\mathrm{II}^{\mathrm{e}}$ siècle av. J.-C., à l'exception de quelques monnaies et lampes impériales qui peuvent être attribuées à une fréquentation domestique ${ }^{12}$, indique un abandon du lieu de culte après cette destruction, plutôt qu'une simple réduction de l'espace du sanctuaire.

9. Humfry Payne et alii, Perachora. The Sanctuaries of Hera Akraia and Limenia, t. 1 : Architecture, Bronzes, Terracottas, Oxford, Clarendon Press, 1940, p. 14-15 et 93 ; John Coulton, "The West Court at Perachora », The Annual of the British School at Athens, t. 62, 1967, p. 353-371, en particulier p. 363-364 et 370-371.

10. Charles K. Williams, III, "Corinth 1977, Forum Southwest », Hesperia, t. 47 , fasc. 1, 1978, p. 1-39, ici p. 5-12.

11. John Coulton, The West Court, p. 370-371; John Coulton, « The Stoa by the Harbour at Perachora », The Annual of the British School at Athens, t. 59, 1964, p. 100-131, ici p. 131.

12. Thomas Dunbabin et alii, Perachora. The Sanctuaries of Hera Akraia and Limenia, t. 2 : Pottery, Ivories, Scarabs and Other Objects from the Votive Deposit of Hera Limenia, Oxford, Clarendon Press, 1962, p. 366-367, 391, 399 et 456-460 pour le matériel d'époque impériale ; ibid., p. 364-365 et Humfry Payne et alii, Perachora t. 1, p. 98 et 103 pour le matériel hellénistique. 
Dans d'autres cas, c'est l'espace du temple ou de son autel qui est directement concerné par un changement d'affectation. Ainsi, au lendemain des guerres médiques, un quartier d'habitat est construit à l'emplacement du sanctuaire d'Artémis Kithonè à Milet et des éléments architecturaux de l'ancien temple sont remployés lors de la construction de plusieurs édifices domestiques ${ }^{13}$. Le temple luimême est détruit et partiellement recouvert par une maison. Bien que l'espace situé au sud de ce bâtiment n'ait pas été entièrement fouillé, il est vraisemblablement inclus dans le quartier d'habitat, ce qui semble exclure un déplacement du temple et de l'autel au sein du même sanctuaire. Le quartier d'habitat est abandonné dès la première partie du $\mathrm{V}^{\mathrm{e}}$ siècle av. J.-C. et, à l'exception d'une vingtaine de fragments de figurines de terre cuite hellénistiques dont la présence ne peut pour l'instant être expliquée ${ }^{14}$, aucun matériel postérieur au $\mathrm{V}^{\mathrm{e}}$ siècle n'a été trouvé à cet endroit. Dès lors, la construction d'une maison au-dessus du temple semble marquer l'abandon du sanctuaire. Dans la même ville, la reconstruction d'une grande maison à péristyle au début du $\mathrm{III}^{\mathrm{e}}$ siècle apr. J.-C. a pour conséquence la destruction partielle du stylobate du temple d'Athén $\mathrm{a}^{15}$ et suggère que la déesse n'est plus honorée à cet endroit. Un autre exemple provient d'Érétrie, où une maison est bâtie au IV siècle av. J.-C. à l'emplacement du «Hérôon de la porte ouest $»^{16}$. Dans le sanctuaire de Poséidon sur l'isthme de Corinthe, ce sont des routes carrossables qui sont construites au-dessus de

13. Michael Kerschner, "Milet 1992-1993. Die Grabung am Kalabaktepe», Archäologischer Anzeiger (dorénavant abrégé AA), 1995, p. 214-220 ; Michael Kerschner et alii, "Die Ostterrasse des Kalabaktepe », AA, 1997, p. 120-122 ; M. Kerschner, «Das Artemisheiligtum auf der Ostterrasse des Kalabaktepe in Milet. Stratigraphie und Keramikfunde der Sondagen des Jahres 1995 », AA, 1999, p. 7-21.

14. Fikret Özcan, «Funde aus Milet XXIV. Hellenistische Terrakotten von der Ostterrasse des Kalabaktepe », AA, 2009, p. 159-165.

15. Armin von Gerkan, Milet, t. I, 8 : Kalabaktepe, Athenatempel und Umgebung, Berlin, Schœtz und Parrhysius, 1925, p. 60-61 et 96-99; Winfried Held, Milesische Forschungen, t. II : Das Heiligtum der Athena in Milet, Mayence, von Zabern, 2000, p. 93.

16. Voir notamment Claude Bérard, Eretria. Fouilles et recherches, t. III : L'Hérôon à la porte de l'ouest, Berne, Francke, 1970, p. 56-58 ; 63-66 ; Karl Reber, Eretria. Ausgrabungen und Forschungen, t. IX : Die klassischen und hellenistischen Wohnhäuser im Westquartier, Lausanne, Payot, 1998, p. 30 ; p. 35-37. 
l'autel ${ }^{17}$, entre la destruction de Corinthe en 146 av. J.-C., qui semble également avoir affecté le sanctuaire, et la refondation du lieu de culte au milieu du $\mathrm{I}^{\mathrm{er}}$ siècle apr. J.-C. Ainsi, bien que la réduction de l'espace d'un sanctuaire ne soit pas nécessairement synonyme de son abandon, la construction, à l'intérieur des limites d'anciens sanctuaires, d'édifices qui ne semblent pas liés au fonctionnement du lieu de culte, et notamment de maisons, est souvent un indice qui suggère que le lieu de culte n'est plus fréquenté.

\section{Des rituels d'abandon}

S'il est possible que certains sanctuaires aient été progressivement délaissés jusqu'à leur abandon définitif, d'autres lieux de culte ne sont plus fréquentés à la suite d'une décision délibérée. Nous pouvons dans ce cas tenter d'identifier des rituels d'abandon. Ainsi, le sanctuaire de San Nicola di Albanella, près de Poséidonia, est recouvert d'une épaisse couche de pierres à la fin du IV ${ }^{\mathrm{e}}$ siècle av. J.-C. ${ }^{18}$. Les quantités importantes de pierres ainsi que l'extension de la couche, qui correspond aux limites du lieu de culte, indiquent qu'il ne s'agit pas d'un dépôt naturel mais d'un acte délibéré destiné à clôturer le sanctuaire. Une couche de pierres semblable semble également avoir recouvert le sanctuaire de Santa Maria d'Anglona, situé près d'Héraclée de Lucanie $^{19}$. Dans d'autres cas, l'identification de rituels d'abandon est moins évidente.

Dans le «Thesmophorion» de Pella, des pierres ne recouvrent pas l'ensemble du sanctuaire, mais uniquement l'autel ${ }^{20}$. Cette

17. Oscar Broneer, Isthmia, t. I : Temple of Poseidon, Princeton, American School of Classical Studies at Athens, 1971, p. 98-101 ; Isthmia, t. II : Topography and Architecture, Princeton, American School of Classical Studies at Athens, 1973, p. 20-22 ; Elizabeth Gebhard et alii, «University of Chicago Excavations at Isthmia, 1989 : III », Hesperia, t. 67, fasc. 4, 1998, p. 405-456.

18. Voir Lorenz Baumer, Mémoires, p. 123-143 (rituels d'abandon) et Maria Cipriani, S. Nicola di Albanella. Scavo di un santuario campestre nel territorio di Poseidonia-Paestum, Rome, Giorgio Bretschneider, 1989, p. 21-28.

19. Helmut Schläger, Ulrich Rüdiger, «Santa Maria d'Anglona (Com. Tursi, Prov. Matera). Rapporto preliminare sulle due campagne di scavi negli anni 1965 e 1966 », Notizie degli scavi di antichità, $8^{\mathrm{e}}$ série, t. 21, 1967, p. 331-353, ici p. 340-342 et 348-353; « Santa Maria d'Anglona (Com. Tursi, Prov. Matera). Scavi nell'anno 1967 », Notizie degli scavi di antichità, $8^{\mathrm{e}}$ série, t. 23, 1969, p. 171-197, ici p. 172-180 et 189-193.

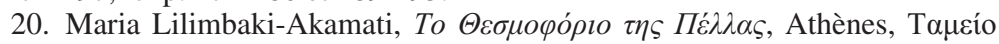

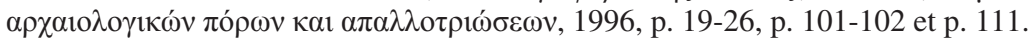


structure est composée de neuf couches de pierres taillées, mêlées à de l'argile. Pourtant, la couche de pierre qui recouvre l'autel est constituée de pierres non taillées, ce qui pourrait peut-être suggérer une fonction différente et indiquer une clôture rituelle du lieu de culte. Dans le sanctuaire du versant est de l'acropole de Pergame, la fouille de deux réservoirs à eau, vraisemblablement utilisés dans le cadre du culte, a révélé l'existence d'une épaisse couche comprenant un riche matériel : ce dernier correspond à la période de fréquentation du sanctuaire, entre le $\mathrm{II}^{\mathrm{e}}$ siècle av. J.-C. et le début du $\mathrm{I}^{\mathrm{er}}$ siècle apr. J.-C. et comprend des éléments d'édifices détruits, ce qui suggère un abandon à la suite d'une destruction violente, peut-être due à un séisme. Ces couches semblent sceller les structures hydrauliques, dont la défonctionnalisation permet vraisemblablement l'identification d'un rituel d'abandon. Le sanctuaire d'Apollon à Soros, en Thessalie, n'a pas, quant à lui, livré de matériel postérieur au début du $\mathrm{III}^{\mathrm{e}}$ siècle av. J.-C., date qui correspond à la fondation de Démétrias et à la disparition probable du site d'habitat dont dépendait le lieu de culte ${ }^{21}$. Dans ce dernier, l'ensemble du matériel de grandes dimensions - notamment des bases de statues, des statues d'enfants et une stèle - a été trouvé dans le pronaos du temple et obstruait l'accès principal à la cella, ce qui peut également être interprété comme une volonté de défonctionnaliser l'édifice religieux au moment de l'abandon. Dans le sanctuaire milésien d'Artémis Kithonè mentionné précédemment, du matériel archaïque de grandes dimensions a été trouvé au niveau des deux rampes qui permettaient l'accès à la terrasse du sanctuaire. Au nord-ouest, il s'agit d'un relief et d'un perirrhanterion archaïques, à l'est d'un relief et d'une statue féminine de la même époque, ainsi que de membres architecturaux qui pourraient provenir du temple archaïque détruit ${ }^{22}$. Le contexte de découverte précis de ces objets n'est connu que dans le cas de la statue féminine, maintenue en place par des pierres dans

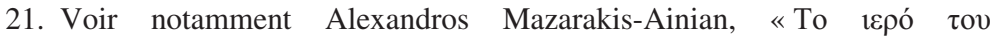

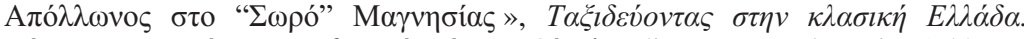

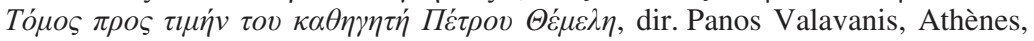

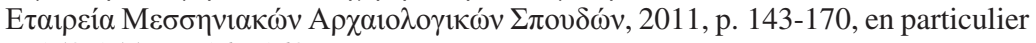
p. $153-155$ et $\mathrm{p}$ 167-168.

22. A. von Gerkan, Milet, p. 10-12 ; M. Kerschner, Kalabaktepe, p. 220 ; Das Artemisheiligtum, p. 14. 
l'angle formé entre le mur de terrassement de la rampe et une marche qui menait au sanctuaire. Il est tentant, dans ce cas également, d'interpréter ces dépôts comme une volonté de fermer symboliquement l'accès au lieu de culte au moment de son abandon.

Si l'interprétation des exemples étudiés est correcte, la diversité des rituels d'abandon rend difficile leur reconnaissance au moment des fouilles. Par ailleurs, la découverte de dépôts qui semblent avoir pour but la défonctionnalisation d'un bâtiment au sein d'un sanctuaire ne permet pas dans tous les cas d'attester l'abandon du lieu de culte. Ainsi, dans le sanctuaire d'Athéna de Francavilla Marittima, en Sybaritide, des centaines d'hydriskai ont été déposées contre la façade et dans le pronaos du bâtiment III, interprété comme le temple principal du sanctuaire. Un tel dépôt, qui semble interdire tout accès à l'édifice, n'a pas été daté mais les hydriskai du sanctuaire ne seraient pas postérieures au $\mathrm{V}^{\mathrm{e}}$ siècle av. J.-C. Or le lieu de culte est encore fréquenté au $\mathrm{IV}^{\mathrm{e}}$ siècle av. J.-C., si bien qu'il pourrait s'agir d'un exemple de rituel d'abandon d'édifice plutôt que de sanctuaire ${ }^{23}$.

\section{Des « faux négatifs » ?}

Les sanctuaires ruraux ou appartenant à des sites d'habitat abandonnés constituent une part importante des sanctuaires antiques abandonnés. Il s'agit souvent, pour cette raison, de sites

23. L'hypothèse d'un rituel d'abandon a été émise par Maria Stoop, « Conjectures on the End of a Sanctuary », Studies in Classical Art and Archaeology. A Tribute to Peter Heinrich von Blanckenhagen, dir. Günter Kopke, Mary Moore, Locust Valley, J.J. Augustin, 1979, p. 179-183, contra Marianne Kleibrink et alii, «Water for Athena: Votive Gifts at Lagaria (Timpone della Motta, Francavilla Marittima, Calabria)», World Archaeology, t. 36, fasc. 1, p. 43-67, ici p. 64, n. 7. Pour la datation des hydriskai, voir par exemple Maria Gentile et alii, « Il santuario sul Timpone Motta di Francavilla Marittima (CS) : nuove prospettive di ricerca dall'analisi dei vecchi scavi », Depositi votivi e culti dell'Italia antica dall'età arcaica a quella tardo-repubblicana, dir. Annamaria Comella, Sebastiana Mele, Bari, Edipuglia, 2005, p. 651-668, ici p. 655-657 et fig. 2b. Pour l'existence de matériel du IV siècle, voir par exemple Marianne Maaskant-Kleibrink, «Religious Activities on the "Timpone della Motta", Francavilla Marittima - and the Identification of Lagaría », Babesch, t. 68, 1993, p. 1-47, ici p. 44-45, et Maria Tommasa Granese, "Culto e pratiche rituali nel santuario arcaico di Francavilla Marittima (Sibari - CS) », Annuario della Scuola archeologica di Atene ser. III, 6, 1, 84, p. 417-464, ici p. 449-450. 
qui n'ont jamais été réoccupés dans l'Antiquité et pour lesquels aucun changement d'affectation ne peut être constaté. Par ailleurs, des rituels d'abandon ne sont que rarement identifiés, en raison notamment de perturbations stratigraphiques postérieures, ainsi que du caractère très récent de la recherche concernant ce phénomène, tandis qu'il est possible que certains sanctuaires aient été abandonnés progressivement, sans que de tels rituels aient été organisés. Dès lors, l'absence de matériel postérieur à une certaine date représente dans la plupart des cas l'unique indice de l'abandon d'un sanctuaire. Or de nombreux lieux de culte ne sont connus que par des prospections de surface. C'est par exemple le cas des sanctuaires de la ville de Latmos, qui semblent avoir été abandonnés au même moment que le site d'habitat, à la fin du IV siècle av. J.-C ${ }^{24}$. D'autres sanctuaires ont fait l'objet de campagnes de fouilles brèves et parfois anciennes. Ainsi, le sanctuaire de Zeus Akraios au sommet du Pélion, qui n'a pas fourni de matériel postérieur au début du III $^{\mathrm{e}}$ siècle av. J.-C., a été fouillé en une unique campagne d'une dizaine de jours au début du $\mathrm{XX}^{\mathrm{e}}$ siècle ${ }^{25}$. Une étude plus approfondie est nécessaire pour établir l'histoire d'un lieu de culte, comme l'indique le cas du sanctuaire d'Héra à Délos : l'unique matériel mis au jour lors des premières fouilles, sous la cella du temple, était daté de l'époque archaïque ${ }^{26}$ et ce n'est que près d'un siècle plus tard que la reprise des travaux a permis la découverte de quantités importantes de céramique hellénistique ${ }^{27}$.

En outre, des facteurs humains et naturels peuvent causer la disparition de couches archéologiques et de toute trace de fréquentation postérieures à une époque donnée. Les fouilles du

24. Anneliese Peschlow-Bindokat, Feldforschungen im Latmos. Die karische Stadt Latmos, Berlin, De Gruyter, 2005, p. 23-29.

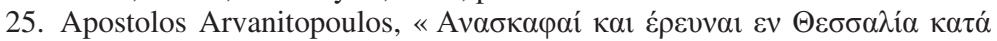

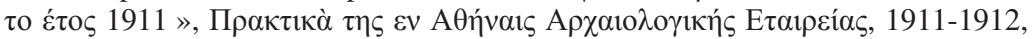
p. 280-356, ici p. 305-312.

26. André Plassart, Exploration archéologique de Délos, t. XI : Les sanctuaires et les cultes du Mont Cynthe, Paris, De Boccard, 1928, p. 149-174.

27. Voir James Whitley, «Archaeology in Greece 2002-2003 », Archaeological Reports, t. 49, 2002-2003, p. 1-88, ici p. 73-74; «Archaeology in Greece 2003-2004 », Archaeological Reports (dorénavant abrégé AR), t. 50, 2003-2004, p. 1-92, ici p. 70 ; «Archaeology in Greece 2004-2005», AR, t. 51, 2004-2005, p. 1-118, ici p. 95 ; J. Whitley et alii, «Archaeology in Greece 2006-2007 », AR, t. 53, 2006-2007, p. 1-121, ici p. 84. 
sanctuaire de Némée ont par exemple montré la destruction violente de la plupart de ses édifices, dont le temple de Zeus, à la fin du $v^{\mathrm{e}}$ siècle av. J.-C. ${ }^{28}$. Le lieu de culte n'a livré aucun matériel correspondant aux décennies suivantes et ne semble avoir été reconstruit que dans le dernier tiers du IV siècle av. J.-C. Pourtant, les concours néméens sont attestés pendant cet intervalle et une inscription d'Épidaure datée d'environ 370 av. J.-C. mentionne l'envoi de messagers à Némée ${ }^{29}$. L'absence d'infrastructures permettant l'organisation des concours ainsi que de tout matériel archéologique exclut la possibilité que de tels événements aient pu être organisés de façon régulière dans le sanctuaire pendant la majeure part du $\mathrm{IV}^{\mathrm{e}}$ siècle ; ils pourraient avoir eu lieu à Argos ou à Kléonai à cette époque ${ }^{30}$. Le sanctuaire était-il quant à lui toujours fréquenté ? À l'époque impériale, le nom de Némée désigne le sanctuaire argien dans lequel les concours sont désormais organisés ${ }^{31}$. Il est possible, de la même façon, que les références écrites à Némée au $\mathrm{IV}^{\mathrm{e}}$ siècle av. J.-C. ne désignent pas le sanctuaire étudié mais le lieu où se déroulent provisoirement les concours. Il ne faut toutefois pas négliger le fait que l'activité agricole de l'Antiquité tardive dans le secteur du temple et de son autel est responsable d'une forte perturbation de la stratigraphie. Des couches archéologiques du $\mathrm{VI}^{\mathrm{e}}$ siècle apr. J.-C. se retrouvent ainsi directement au-dessus de couches de la fin du IV siècle av. J.-C. voire de l'Helladique Récent ${ }^{32}$. Dans ce contexte, une

28. Pour les résultats des fouilles, voir notamment Excavations at Nemea: Topographical and Architectural Studies : the Sacred Square, the Xenon, and the Bath, Berkeley, University of California Press, 1992 ; Stephen Miller, Excavations at Nemea, t. II : The Early Hellenistic Stadium, Berkeley, University of California Press, 2001. Pour un état des lieux du sanctuaire au IV siècle, voir Manuela Mari, «Festa mobile. Nemea e i suoi giochi nella tradizione letteraria e nell'evidenza materiale I. L'età arcaica e classica », Incidenza dell'Antico, t. 6, 2008, p. 91-132.

29. Concours néméens : I. Agon. 25, 1. 4. Inscription d'Épidaure : IG IV² 102, A II, 1. 114-115. Voir aussi Démosthène, Contre Midas, XXI, 115.

30. Pour l'hypothèse d'un transfert à Argos, voir par exemple Stephen Miller, « Excavations at Nemea 1977 », Hesperia, t. 47, fasc. 1, 1978, p. 58-88, ici p. 83; pour celle d'un transfert à Kléonai, voir Jean-Yves Strasser, "Argos, Kléonai et les Néméa. À propos de $I G \mathrm{II}^{2}, 365$ », Épire, Illyrie, Macédoine... Mélanges offerts au Professeur Pierre Cabanes, dir. Danièle Berranger-Auserve, ClermontFerrand, Presses Universitaires Blaise Pascal, 2007, p. 329-347, ici p. 331 et n. 14.

31. Voir par exemple M. Mari, Festa mobile, p. 123-124.

32. Stephen Miller, «Excavations at Nemea 1978 », Hesperia, t. 48, fasc. 1, 1979, p. 73-103, ici p. 81 ; «Excavations at Nemea 1973-1974», Hesperia, t. 44, fasc. 2, 1975, p. 143-172, ici p. 158. 
disparition du matériel lié à une activité minimale qui se serait maintenue au IV siècle av. J.-C. autour de l'autel n'est pas exclue.

De la même façon, le Panionion, situé près de Güzelçamlı au pied du mont Mycale, et identifié grâce à des inscriptions du $\mathrm{IV}^{\mathrm{e}}$ siècle av. J.-C., n'a livré aucun matériel postérieur à cette date et seulement une quantité très faible de matériel archaïque, malgré son importance majeure, dont témoigne notamment Hérodote ${ }^{33}$. Hans Lohmann a pour cette raison suggéré que le Panionion était à l'origine situé à un autre emplacement à l'époque archaïque et qu'il avait été transféré près de Güzelçamlı au cours du IV siècle av. J.-C. Peu après, le sanctuaire aurait une nouvelle fois été transféré à Priène ${ }^{34}$. Pourtant, Strabon écrit que le Panionion est situé près du détroit de $\operatorname{Samos}^{35}$, ce qui correspond à la situation du Panionion de Güzelçamlı et suggère que le sanctuaire se trouve encore au même emplacement au début de l'époque impériale. La colline sur laquelle est situé le lieu de culte a reçu son nom actuel d'Otomatik Tepe en raison de son utilisation militaire dans le premier quart du $\mathrm{xx}^{\mathrm{e}}$ siècle, qui pourrait être à l'origine de la disparition de toute trace d'activité multiséculaire à cet endroit ; par ailleurs, la présence de deux fours à chaux à proximité peut expliquer la disparition d'une partie du matériel, et notamment des inscriptions et des statues ${ }^{36}$.

D'autres transformations post-dépositionnelles sont indirectement liées à l'activité humaine. Ainsi, à Corinthe, l'extraction de calcaire à proximité du sanctuaire d'Apollon expliquerait la découverte de matériel archaïque en contrebas du temple ${ }^{37}$.

33. I.Priene 2, 398-399; Hérodote, I, 141 ; 148 ; 170 ; VI, 7. Pour le matériel du sanctuaire, voir Gerhard Kleiner et alii, Panionion und Melie, Berlin, de Gruyter, 1967, notamment p. 16 et p. 75-76.

34. Hans Lohmann, «Melia, das Panionion und der Kult des Poseidon Helikonios », Neue Forschungen zu Ionien. Fahri Işik zum 60. Geburtstag gewidmet, dir. Elmar Schwertheim, Engelbert Winter, Bonn, Rudolf Habelt, 2005, p. 57-91 et «Ionians and Carians in the Mycale. The Discovery of Carian Melia and the Archaic Panionion », Landscape, Ethnicity and Identity in the Archaic Mediterranean Area, dir. Gabriele Cifani, Simon Stoddart, Oxford, Oxbow Books, 2012, p. 32-50. La majeure partie des arguments du chercheur a été réfutée par Alexander Herda, «Panionion - Melia, Mylakessos - Mykale, Perseus und Medusa », Istanbuler Mitteilungen, t. 56, 2006, p. 43-102, notamment p. 53-54.

35. Strabon, XIV, 1, 20 (C 639).

36. G. Kleiner et alii, Panionion, p. 23 et 21.

37. Nancy Bookidis, Ronald Stroud, «Apollo and the Archaic Temple at Corinth », Hesperia, t. 73, fasc. 3, 2004, p. 401-426, ici p. 422-423. 
Cependant, la disparition de couches archéologiques a souvent une origine naturelle. L'action des vagues peut ainsi entraîner la destruction partielle de sanctuaires situés près d'un rivage, comme dans le cas d'un bâtiment interprété comme un temple, au bord du lac Paralimni en Béotie, ou du sanctuaire hellénistique B6 sur l'île d'Ikaros - l'actuelle Failaka au Koweït ${ }^{38}$. Dans les terrains en pente, la pédoturbation, le mouvement des sols, peut s'expliquer par le ruissellement des eaux de pluie, des glissements de terrain mais aussi par l'action des animaux et des végétaux ${ }^{39}$. En Laconie, près du cap Malée, des vases miniatures ont par exemple été découverts en bas d'une pente à la suite d'un épisode pluvieux et ont permis de supposer l'existence d'un sanctuaire en amont ${ }^{40}$. Les contraintes liées à la recherche archéologique, notamment d'ordre budgétaire, ne permettent pas, dans tous les cas, une fouille exhaustive des lieux de culte étudiés accompagnée d'une prospection des environs, si bien que de nombreuses fouilles peuvent produire des « faux négatifs ».

\section{Le contexte historique}

Si l'identification d'un sanctuaire isolé qui semble être abandonné demeure, dans bien des cas, incertaine, la découverte de nombreux lieux de culte dont la fréquentation semble cesser à la même époque et au sein d'une même région rend l'hypothèse d'un abandon plus probable et permet de chercher des causes communes à la désaffectation de ces sanctuaires. Susan Alcock note ainsi la baisse du nombre de sanctuaires ruraux dans diverses régions du monde grec aux époques hellénistique et impériale. Cette baisse pourrait s'expliquer par un exode rural ainsi que par l'importance prise par l'évergétisme privé dans le financement des activités

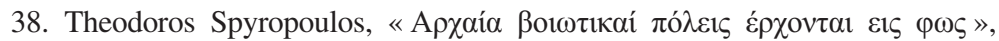

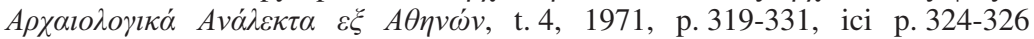
(Paralimni); Annie Caubet, Jean-François Salles, «Le sanctuaire hellénistique (B 6) », Falaika. Fouilles françaises. 1983, dir. Jean-François Salles, Lyon, Maison de l'Orient et de la Méditerranée Jean Pouilloux, 1984, p. 73-156, ici p. 73 (Ikaros).

39. Voir par exemple Raymond Wood, Donald Johnson, «A Survey of Disturbance Processes in Archaeological Site Formation», Advances in Archaeological Method and Theory, t. 1, 1978, p. 315-381.

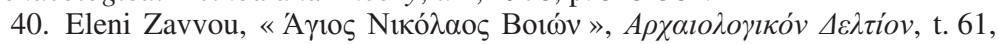
B'1, 2006, p. 303. 
rituelles et des constructions, qui se concentrent dans quelques sanctuaires seulement, au détriment des lieux de culte mineurs. Lorenz Baumer propose quant à lui de mettre en lien l'abandon de nombreux lieux de culte attiques à partir de l'époque hellénistique avec un affaiblissement des dèmes ${ }^{41}$. De même, comme nous l'avons évoqué précédemment, les lacunes dans la documentation archéologique des sanctuaires de l'isthme de Corinthe et de Pérachora, à partir du $\mathrm{II}^{\mathrm{e}}$ siècle av. J.-C, peuvent être expliquées par la destruction de Corinthe en - 146 et l'abandon - provisoire dans le premier cas, définitif dans le second - des sanctuaires extra-urbains de la cité.

L'abandon de sanctuaires urbains et suburbains semble également fréquent à la suite d'abandons de sites d'habitat. Il s'agit notamment de synécismes - lorsque plusieurs sites d'habitat sont abandonnés et que leur population est réunie au sein d'une même ville - et de métécismes - lorsqu'une ville entière est déplacée à un nouvel emplacement. Nous avons mentionné le sanctuaire d'Apollon à Soros, vraisemblablement abandonné au moment de la fondation de Démétrias par synécisme, ainsi que le cas de Latmos, dont les espaces interprétés comme des sanctuaires n'ont livré aucun matériel postérieur au IV ${ }^{\mathrm{e}}$ siècle av. J.-C.42. De la même façon, à Smyrne, le sanctuaire d'Athéna ne semble pas avoir été fréquenté après le déplacement de la ville à la même époque ${ }^{43}$. Le nombre d'exemples disponibles est certes très faible en raison de la rareté des études archéologiques qui portent à la fois sur les sites d'habitat et les sanctuaires. Cependant, la découverte de matériel attestant l'existence d'un culte à Néandreia, un siècle après l'abandon de la ville lors du synécisme d'Alexandrie de Troade $^{44}$, demeure une exception. Il est probable que l'abandon de sites d'habitat ait la plupart du temps entraîné l'abandon de leurs sanctuaires.

41. Voir respectivement S.E. Alcock, «Minding the Gap», en particulier p. 259-260 et L. Baumer, Mémoires, p. 61-78.

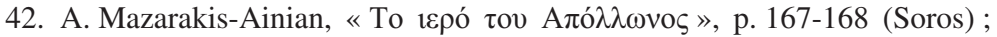
A. Peschlow-Bindokat, Feldforschunge, p. 23-29 (Latmos).

43. John Cook, Richard Nicholls, Old Smyrna Excavations. The Temples of Athena, Londres, British School at Athens, 1998, p. 181-185.

44. Axel Filges, Petra Matern, «Eine Opfergrube der Demeter in Neandria », Die Troas. Neue Forschungen zu Neandria und Alexandria Troas II, dir. Elmar Schwertheim, Hans Wiegartz, Bonn, Rudolf Habelt, 1996, p. 43-86. 
Pourtant, des incertitudes concernant la fréquentation de certains lieux de culte peuvent demeurer même lorsque le contexte suggère un abandon. C'est ce que souligne l'exemple d'un petit sanctuaire de Mégalopolitide, situé près des villes de Trapézonte et de Basilis et qui n'a pas livré de matériel postérieur à la fin IV siècle av. J.-C.45. Le site de Kyparissia, qui correspond à l'un de ces deux sites d'habitat, semble avoir été abandonné à la même époque, quelques décennies après le synécisme de Mégalopolis ${ }^{46}$. Un abandon contemporain du sanctuaire semble a priori vraisemblable. Pourtant, le lieu de culte semble correspondre au sanctuaire de Bathos que Pausanias, cinq siècles plus tard, décrit en utilisant le présent ${ }^{47}$. Si cette identification est correcte, plusieurs facteurs pourraient expliquer l'absence de matériel correspondant à cinq siècles de fréquentation, notamment la brièveté des fouilles anciennes et le pillage du site au XIX ${ }^{\mathrm{e}}$ siècle. Par ailleurs, le matériel mis au jour par Klon Stephanos au début du Xx $x^{\mathrm{e}}$ siècle n'a pas été daté. Dès lors, malgré l'abandon apparent du site d'habitat de Kyparissia à la fin du IV ${ }^{\mathrm{e}}$ siècle av. J.-C, les informations dont nous disposons ne sont pas suffisantes pour conclure de façon certaine à un abandon du lieu de culte à cette date.

\section{LES TRANSFERTS DE CULTES}

Nous avons vu la difficulté à identifier archéologiquement l'abandon d'un sanctuaire. Mais, lorsque les fouilles se déroulent dans des conditions satisfaisantes et qu'un tel abandon est vraisemblable, est-il possible de conclure à la disparition du culte de la ou des divinités honorées? Deux inscriptions révèlent que certains cultes peuvent être transférés à un nouvel emplacement. Dans la première, la cité de Tanagra consulte un oracle pour savoir

45. A.G. Bather, V. Yorke, «Excavations on the Probable Sites of Basilis and Bathos", Journal of Hellenic Studies, t. 13, 1892-1893, p. 227-231, ici

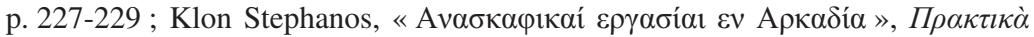

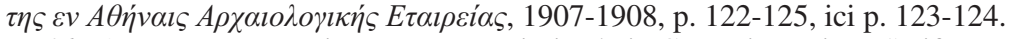

46. Anna Karapanagiotou, «Kyparissia bei Gortynia: eine Steifenstadt klassischer Zeit im westlichen Arkadien », Neue Forschungen zu griechischen Städten und Heiligtümern. Festschrift für Burkhardt Wesenberg, dir. Heide Frielinghaus, Jutta Stroszeck, Möhnesee, Bibliopolis, 2010, p. 127-138.

47. Pausanias, Periégèse, VIII, 29, 1. 
s'il est préférable de laisser le sanctuaire de Déméter et Koré à son emplacement d'origine ou de le transférer dans la ville ou à un autre endroit ${ }^{48}$. La seconde inscription, malheureusement lacunaire, concerne le transfert du sanctuaire de la Sôteira sur l'île d'Ikaros, dans le golfe persique ${ }^{49}$. Une lamelle oraculaire de Dodone et une inscription de Skopélos, souvent mises en lien avec ces deux textes, pourraient quant à elles mentionner des déplacements au sein d'un sanctuaire plutôt que des transferts de cultes, puisque le déplacement concerne le temple, ou l'ensemble formé par le temple, la statue de culte et l'autel, et non le sanctuaire lui-même ${ }^{50}$. Les causes des deux transferts attestés par l'épigraphie sont inconnues. Toutefois, Pline le Jeune nous informe de la volonté des Nicomédiens de déplacer un temple pour des raisons urbanistiques lors de la construction d'un nouveau forum ${ }^{51}$.

Les déplacements de population peuvent également expliquer certains transferts de cultes. Les métécismes n'entraînent vraisemblablement pas de rupture dans la tradition religieuse des cités concernées. Or, comme nous l'avons mentionné précédemment, les sanctuaires des sites d'habitat abandonnés ne semblent plus fréquentés par la suite, si bien qu'il faut supposer un transfert de leurs cultes dans le nouveau site d'habitat. Ainsi, Athéna, qui semble avoir été une divinité majeure dans l'ancienne ville de Latmos, occupe également un rôle central dans la ville d'Héraclée du Latmos qui lui succède ${ }^{52}$. Lors de synécismes, plusieurs

48. Léopold Migeotte, Les souscriptions publiques dans les cités grecques, Genève, Droz, 1992, p. 75-76, nº 28.

49. Voir par exemple Charlotte Roueché, Susan Sherwin-White, «Some Aspects of the Seleucid Empire : the Greek Inscriptions from Failaka, in the Arabian Gulf », Chiron, t. 15, 1985, p. 1-39, n 3 , 1. 7-16. Sur ces deux inscriptions, voir notamment Séverine Quantin, François Quantin, « Le déplacement du temple d'Athéna Polias en Chaonie. Remarques sur les cosiddetti "temples voyageurs" », Épire, Illyrie, Macédoine... Mélanges offerts au Professeur Pierre Cabanes, dir. Danièle Berranger-Auserve, Clermont-Ferrand, Presses Universitaires Blaise Pascal, 2007, p. 175-196, ici p. 182-184 et p. 186-187, avec la bibliographie antérieure.

50. Éric Lhôte, Les lamelles oraculaires de Dodone, Genève, Droz, 2006, n 11 (Dodone); IG XII, 8, 640 (Skopélos). À ce sujet, voir par exemple S. et F. Quantin, ibid., en particulier p. 176-185.

51. Pline, Lettres, X, 49. Voir aussi Dion Chrysostome, Discours, XLVII, $16-17$

52. Athéna à Latmos: Michael Wörrle, «Inschriften von Herakleia am Latmos III », Chiron, t. 33, 2003, p. 121-143, 1. 33-36. Athéna à Héraclée : M. Wörrle, «Inschriften von Herakleia am Latmos II. Das Priestertum der 
communautés sont regroupées au sein d'une nouvelle cité, dont le panthéon comprend de nouvelles divinités qui permettent de fédérer l'ensemble de la population, mais également certaines divinités honorées précédemment par les communautés qui prennent part à ce processus ${ }^{53}$. Dès lors, il est vraisemblable que l'abandon de sites d'habitat lors de synécismes entraîne, comme dans le cas des métécismes, le transfert de certains cultes dans la nouvelle ville.

De tels transferts peuvent être identifiés lorsque l'abandon d'un sanctuaire est confirmé mais que le culte de la divinité continue à être attesté. C'est le cas du culte d'Artémis Kithonè à Milet, dont le sanctuaire, comme nous l'avons vu, est détruit et remplacé par un quartier d'habitat à la suite des guerres médiques : le culte de la déesse est attesté entre le $\mathrm{III}^{\mathrm{e}}$ siècle av. J.-C. et le III $^{\mathrm{e}}$ siècle apr. J.-C. et une inscription du $\mathrm{I}^{\mathrm{er}}$ siècle av. J.-C. mentionne explicitement son temple $e^{54}$. Il est probable que son culte ait été transféré dans la ville basse dès le $\mathrm{v}^{\mathrm{e}}$ siècle av. J.-C., ou du moins refondé à un nouvel emplacement à l'époque classique ou au début de l'époque hellénistique. En l'absence de sources épigraphiques ou littéraires, la reconnaissance archéologique de transferts de cultes est plus difficile. Nous avons mentionné la ville de Néandreia, abandonnée à la fin du IV siècle av. J.-C. à la suite de la fondation d'Alexandrie de Troade. Son temple principal, daté de l'époque archaïque et dédié à Apollon, est détruit par un incendie à une époque indéterminée et n'est jamais reconstruit ${ }^{55}$. Il est donc possible qu'il n'ait pas été fréquenté à l'époque hellénistique. Or l'iconographie des premières monnaies de la nouvelle polis reprend celle des monnaies de Néandreia qui re-

Athena Latmia », Chiron, t. 20, 1990, p. 19-58, inscription S II A, 1. 4-5 ; Milet I, 3, 150, 1. 119-120.

53. Voir notamment Robert Parker, «Subjection, Synœcism and Religious Life », The Politics of Ethnicity and the Crisis of the Peloponnesian League, dir. Peter Funke, Nino Luraghi, Cambridge Mass., Center for Hellenistic Studies, 2009, p. 183-214, en particulier p. 192-195.

54. Callimaque, Hymne à Artémis, v. 225-226; Milet VI, 1, 202, 1. 1-4 ( ${ }^{\text {er }}$ siècle av. J.-C.) ; I. Didyma 315, 1. 1-2 (III ${ }^{\mathrm{e}}$ siècle apr. J.-C.).

55. Robert Koldewey, Neandria. Einundfünfzigstes Programm zum Winckelmannsfeste der archäologischen Gesellschaft zu Berlin, Berlin, Georg Reimer, 1891, en particulier p. 30. Elmar Schwertheim, «Die Inschriften», Neue Forschungen zu Neandria und Alexandria Troas, dir. E. Schwertheim, H. Wiegartz, Bonn, Rudolf Habelt, 1994, p. 39-47, ici p. 40-41, nº 2. 
présentent Apollon ${ }^{56}$, ce qui pourrait suggérer la continuité du culte du dieu de l'ancienne ville au sein de la nouvelle polis. De plus, un temple non attribué d'Alexandrie de Troade est orienté vers le site de Néandreia : l'édifice pourrait être dédié à l'Apollon de cette ville, et son orientation indiquerait dans ce cas la volonté de créer un lien visuel entre l'ancien lieu de culte et son successeur ${ }^{57}$. Bien que ces indices soient extrêmement ténus, un transfert de culte demeure plausible puisque Néandreia est la principale communauté qui prend part au synécisme d'Alexandrie de Troade jusqu'à l'inclusion d'Hamaxitos et de son Smintheion au II $^{\mathrm{e}}$ siècle av. J.-C. ${ }^{58}$.

La rareté des sources concernant des transferts de cultes suggère $a$ priori qu'il s'agit d'un phénomène très rare ${ }^{59}$. Nous avons pourtant vu l'écart qui demeure entre le faible nombre de sources écrites relatant des abandons de sanctuaires et l'important nombre de lieux de culte vraisemblablement abandonnés d'après les fouilles. La fréquence des transferts de culte est dès lors difficile à évaluer : en raison de sa difficile reconnaissance archéologique, ce phénomène pourrait avoir été largement sous-estimé, étant donné qu'il était vraisemblablement courant lors des déplacements de population. Du reste, des transferts pouvaient avoir lieu à d'autres occasions, notamment lors de réaménagements urbains.

\section{CONCLusion}

Malgré le faible intérêt suscité jusqu'à récemment par le sujet, l'abandon de sanctuaires ne constitue pas un phénomène marginal. D'après un rapide décompte dans la base de données qui recense

56. Andrew Meadows, «The Earliest Coinage of Alexandria Troas », Numismatic Chronicle, t. 164, 2004, p. 47-70, ici p. 52-55.

57. Daniela Pohl, «Der dorische Tempel von Alexandria Troas. Überlegungen zu Rekonstruktion und Datierung », Die Troas. Neue Forschungen III, dir. E. Schwertheim, Bonn, Rudolf Habelt, 1999, p. 85, 93, en particulier p. 85 et 90 .

58. Voir Alain Bresson, «Hamaxitos en Troade», Espaces et pouvoirs dans l'Antiquité, de l'Anatolie à la Gaule, dir. Julie Dalaison, Grenoble, CRHIPA, 2007, p. 139-158, ici p. 150-156.

59. Outre les inscriptions mentionnées et le texte de Pline, voir aussi Strabon, Géographie, V, 4, 5 (C 244-245) ; VII, 7, 12 (C 329) ; IX, 2, 10 (C 404) ; XIII, 1, 63 (C 612-613), qui suppose toutefois des transferts de cultes probablement sans correspondance avec des événements historiques. 
les sanctuaires ruraux de Grèce, créée par Lorenz Baumer en $2006^{60}$, environ $70 \%$ des quelque 125 sanctuaires pour lesquels des indications chronologiques relativement précises sont disponibles pourraient avoir été abandonnés, et ce entre l'époque archaïque et le début de l'époque impériale. Pourtant, dans de nombreux cas, les conditions de fouilles ne permettent pas d'établir l'abandon avec un degré de certitude satisfaisant. Même lorsque l'abandon est confirmé, il n'offre aucune indication sur le sort du culte, qui pourrait avoir été transféré à un autre emplacement, puisque l'absence de preuve d'un tel transfert ne peut constituer la preuve de son absence. Dès lors, les sources archéologiques ne peuvent en aucun cas attester la disparition d'un culte ${ }^{61}$.

alaya.palamidis@alumni.uliege.be

60. www.kultundraum.net (consulté le 3 février 2017).

61. Je tiens à remercier Karin Mackowiak et Christian Stein de m'avoir donné l'opportunité de présenter une partie de mes recherches de doctorat portant sur l'abandon de sanctuaires et le transfert de cultes en Grèce antique. 